\title{
Bioseguridad y manejo de la vía aérea en el paciente pediátrico con infección por SARS-CoV-2
}

\section{Biosecurity and airway management in the pediatric patient with SARS-CoV-2 infection}

\author{
Adriana del Carmen Luna-Castañeda, ${ }^{*}$ Alejandra Sánchez-González,
}

Alicia Buenrostro-Gaitán, ${ }^{\S}$ Alma Karina Bernardino-González, ${ }^{\S}$ Ana Karen Gutiérrez-Bautista, ${ }^{*}$ Ángel Flores-Martínez, ${ }_{1}^{\S}$ Areli Pichardo-Estrada,* Blanca Estela Martínez-Martínez,* Brenda Aguilar-Viveros,* Calixto Galeana-Miramontes,, Carlos Juárez-Ortiz, ${ }^{\S}$ Carlos Ramos-Verástica, ${ }^{*}$ Claudia Serine Pestaña-Fonseca, ${ }^{\$}$ Claudia Quezada-López, Elizabeth Hernández-Alvídrez, Esmeralda Salazar-Reséndiz, Eunice Nava-Hernández," Ingrid Basemat Guerrero-Macías, ${ }^{*}$ Irasema Rodríguez-Godínez, Irma Patricia Pérez-Vieyra, ${ }^{\$}$ Karen Shantal Trejo-Rivera, ${ }^{* *}$ Laura Patricia Thomé-Ortiz,* Luis Fernando Torres-Pedraza, ${ }^{\$}$ María del Rocío García-Olvera, ${ }^{\ddagger \ddagger}$ María Viridiana Figueroa-Gómez, ${ }^{*}$ Nancy Ojeda-Luna, ${ }^{\$ \S}$ Martha Hervert-Jonguitud, ${ }^{\S}$ Montserrat Hidalgo-Vargas," Silvia Graciela Moysén-Ramírez, Vania Zamara Preciado-Sánchez, ${ }^{\S}$ Violeta Martínez-Alcántara, ${ }^{\S}$ Yerania Sánchez-Miranda ${ }^{\S}$

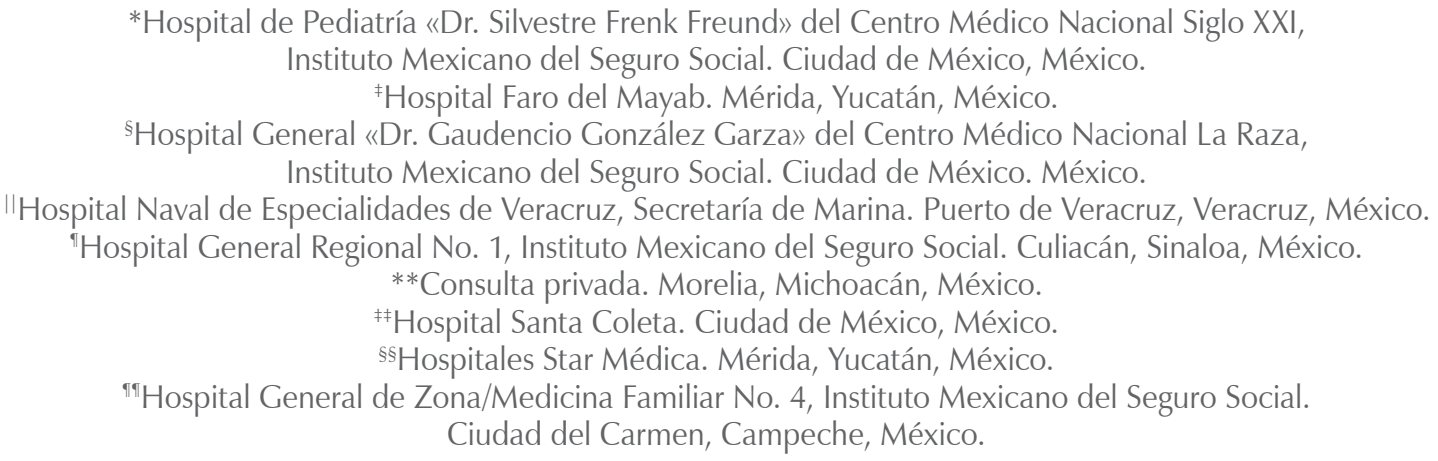

RESUMEN. De acuerdo con la Secretaría de Salud de México, el primer caso confirmado de COVID-19 (SARS-CoV-2) en nuestro país ocurrió el 27 de febrero de 2020. Desde su llegada, el personal dedicado a la atención pediátrica ha desarrollado estrategias de capacitación con la finalidad de atender a sus pacientes y ser parte del personal de respuesta
ABSTRACT. The first confirmed case of COVID-19 (SARS-CoV-2) in our country occurred on February 27, 2020, according to the Mexican Ministry of Health. The staff engaged in pediatric care has developed training strategies to serve their patients and to be part of the pandemic response staff. Learning biosecurity mechanisms is significant to prevent

Correspondencia:

Dr. Carlos Juárez-Ortiz

Neumología Pediátrica. Hospital General «Dr. Gaudencio González Garza» del Centro Médico Nacional La Raza,

Instituto Mexicano del Seguro Social. Ciudad de México, México.

Correo electrónico: broncoscopiajuarez@gmail.com

Trabajo recibido: 16-VI-2020; aceptado: 23-VII-2020.

Citar como: Luna-Castañeda AC, Sánchez-González A, Buenrostro-Gaitán A, Bernardino-González AK, Gutiérrez-Bautista AK, Flores-Martínez A. Bioseguridad y manejo de la vía aérea en el paciente pediátrico con infección por SARS-CoV-2. Neumol Cir Torax. 2020; 79 (4): 265-277. https://dx.doi. org/10.35366/97970 
ante la pandemia. El aprendizaje de los mecanismos de bioseguridad resulta significativo para evitar mayor propagación del virus y fortalecer al personal de salud, evitando el contagio. Es por ello que consideramos que los pediatras del país deben estar capacitados para determinar el tipo de equipo de protección personal y las estrategias de protección antes, durante y después de someter a ventilación a los pacientes; asimismo, al realizar maniobras de reanimación cardiopulmonar, conocidas como procedimientos generadores de aerosoles (PGA). Considerando que se prevé una epidemia larga en nuestro país, presentamos este artículo de revisión que sirva como parte del acervo que se requiere para documentar buenas prácticas ante COVID-19.

Palabras clave: Intubación endotraqueal, bioseguridad, vía aérea, COVID-19.

\section{INTRODUCCIÓN}

La Organización Mundial de la Salud (OMS) define el término bioseguridad o seguridad biológica como los principios, técnicas y prácticas aplicadas con el fin de evitar la exposición no intencional a patógenos y toxinas, o su liberación accidental. ${ }^{1}$ Es un conjunto de normas y medidas para proteger al personal de salud frente a los riesgos biológicos químicos y físicos a los que está expuesto en el desempeño de sus funciones, incluyendo a los pacientes y al medio ambiente. ${ }^{2}$

Según los niveles de bioseguridad de acuerdo con los centros para el Control de Enfermedades de Estados Unidos de América (Centers for Disease Control [CDC]), la infección por SARS-CoV-2 se encontraría en el nivel III, es decir, nivel alto de contagiosidad, ya que pertenece a microrganismos altamente patógenos con potencial de transmisión respiratoria, y que pueden provocar una infección grave y potencialmente letal. ${ }^{3}$

Se ha establecido que los mecanismos de transmisión del virus son principalmente por gotas, las cuales tienden a dispersarse hasta distancias de un metro, y no se ha demostrado que éste se transmita por vía aérea a través de partículas suspendidas en el aire. ${ }^{4,5}$ Sin embargo, algunos procedimientos pueden generar aerosoles y, con ello, el riesgo de trasmisión a distancias mayores a dos metros, así como la contaminación de superficies inertes, por lo que el virus puede transmitirse a través del contacto con dichas superficies.

El tema de bioseguridad es un tema de preocupación en situaciones emergentes como la pandemia por SARS-CoV-2. El equipo de protección personal (EPP) es uno de los temas más comentados y emotivos para el personal de salud de primer contacto por dos razones principales: escasez del equipo y su uso inapropiado. ${ }^{6}$

Las recomendaciones para la prevención de infección por aerosoles están principalmente orientadas a áreas críticas donde hay mayor producción de aerosoles y gotas (unidades de terapia intensiva, salas de urgencias, quirófano y salas de labor), en donde se realizan de manera rutinaria procedimientos como: ventilación con presión positiva, as- further spread of the virus and strengthen health care teams with the purpose of preventing contagion. That is why we consider that the pediatricians in Mexico should be trained to determine the type of personal protective equipment and protection strategies before, during and after ventilating patients, also when performing cardiopulmonary resuscitation maneuvers, procedures that are aerosol generators. Considering that a long epidemic is expected in our country, we present this article that serves as part of the knowledge required to document good practices for treating COVID-19.

Keywords: Endotracheal intubation, biosecurity, airway, COVID-19.

piración de vía aérea, intubación endotraqueal, ventilación de alta frecuencia, traqueostomía fisioterapia pulmonar, aerosolterapia y broncoscopia.

De manera general, las medidas principales para disminuir la transmisión son: designación de áreas específicas para la atención de estos pacientes, personal capacitado tanto en la colocación del EPP como en la higiene y limpieza adecuada de las superficies e identificar situaciones con mayor probabilidad de aerosoles que diseminen el virus. ${ }^{8}$

Dentro de las máximas prioridades en el manejo de pacientes en nivel III de bioseguridad se encuentra la seguridad del personal. El personal de salud no debe convertirse en un paciente más ante la premura de otorgar atención, por lo tanto, la colocación del EPP debe ser rigurosamente supervisada por un líder de equipo que se encuentre calificado y entrenado en la bioseguridad. El personal debe estar calificado para este tipo de escenarios, ya que se ha descrito que los EPP pueden modificar el desempeño del personal no entrenado. ${ }^{9}$

El objetivo de este apartado es realizar una descripción del EPP y procedimientos de manejo de vía aérea, para el nivel de bioseguridad III y su uso racional en las diferentes áreas de contacto, principalmente en zonas con mayor probabilidad de contacto por aerosoles.

\section{EQUIPO DE PROTECCIÓN PERSONAL: MANEJO SUGERIDO DEL CUERPO MÉDICO Y DE ENFERMERÍA}

El EPP es una parte fundamental en el manejo del paciente con COVID-19, y brinda protección al trabajador de la salud contra el riesgo de contagio y sus consecuencias. ${ }^{6}$

\section{Manejo general}

El personal que se encuentre en el área de recepción de pacientes, toma de muestras y en área de COVID, debe tener todo el entrenamiento para el uso del EPP, estudiándolo y practicando. Se sugiere que se dividan en equipos, los cuales trabajarán de 4-6 horas de jornada en el área de contención de COVID (Tabla 1). 
Tabla 1: Nivel de protección y equipo de protección personal.

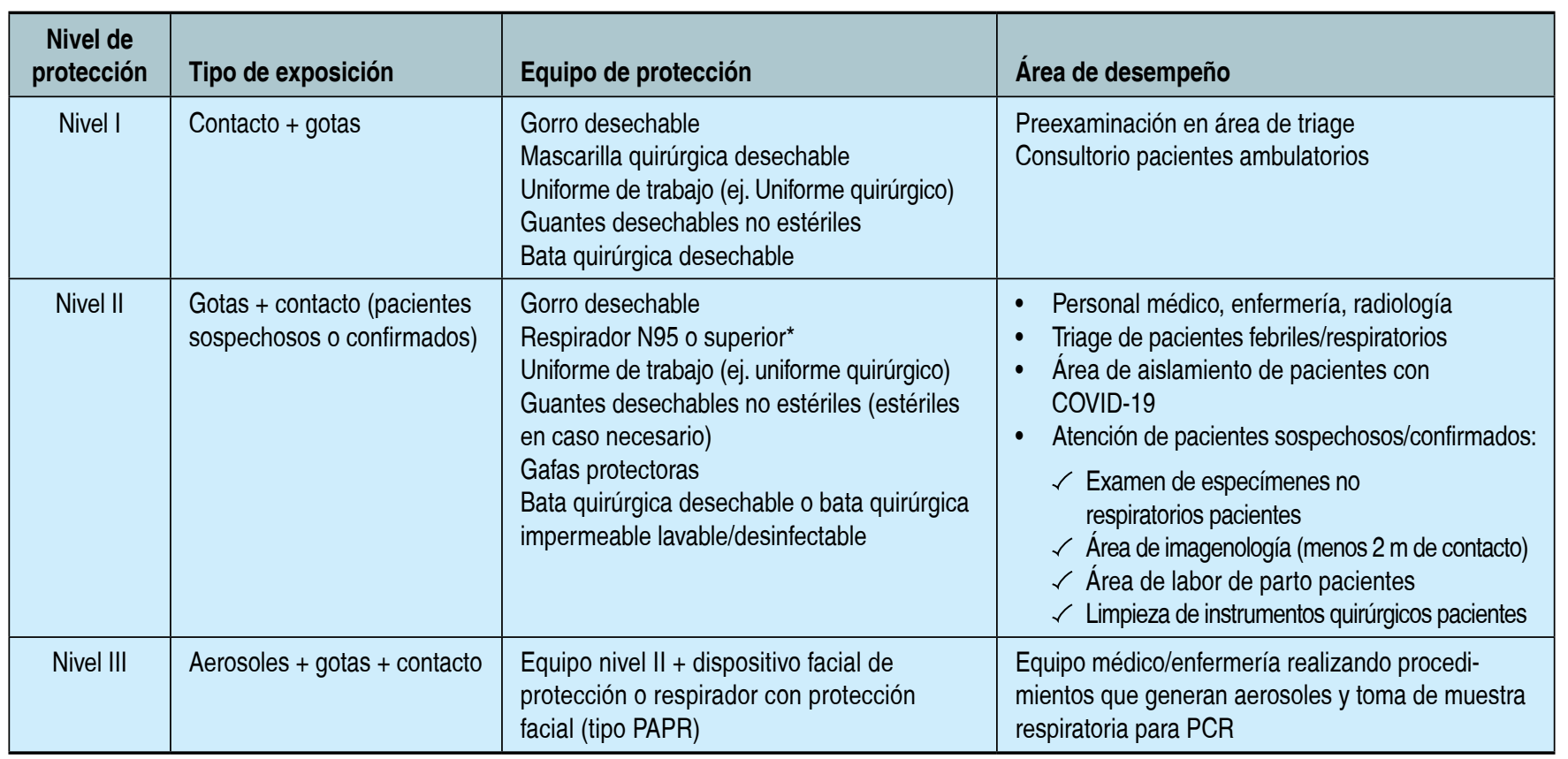

* Las mascarillas N95 son relativamente equivalentes a: FFP2, KN95, P2, Korea 1st class, DS 12.

PAPR = Powered air-purifying respirators; $\mathrm{PCR}=$ reacción en cadena de la polimerasa.

Modificada e integrada de la información de las referencias 10-13.

Personal en contacto con pacientes sospechosos/confirmados a más de 2 metros, deben usar mascarilla quirúrgica y protección ocular, personal de transporte de pacientes sospechosos/confirmados deben llevar: guantes, delantal desechable, mascarilla quirúrgica resistente al agua y gafas protectoras o protección facial. ${ }^{10}$

\section{Equipo de protección respiratoria}

Conociendo los mecanismos de transmisión del SARSCoV-2, es de vital importancia la protección respiratoria en la atención de estos pacientes.

Dentro de la protección respiratoria para el manejo de microorganismos se han descrito cuatro tipos:

a. Mascarillas quirúrgicas y cubrebocas.

b. Respiradores.

c. Respiradores elastoméricos.

d. Respiradores purificadores de aire de tipo forzado (eléctricos) (PAPR por sus siglas en inglés powered airpurifying respirators).

\section{Mascarillas quirúrgicas}

Es un dispositivo médico desechable que protege contra agentes infecciosos transmitidos por «gotas», ya sea de saliva o secreciones del tracto respiratorio superior cuando el usuario exhala. Pueden ser de capa impermeable y dar protección contra salpicaduras de fluidos biológicos o líquidos. Puede ser rígida, flexible o plisada, pueden estar equipados con una visera para protección ocular. Su capacidad de filtración es limitada y no alcanzan el 95\% mínimo requerido para proporcionar protección respiratoria. ${ }^{14}$

\section{Respiradores de partículas simples desechables}

Son equipos que filtran todo el aire que respira el usuario, la eficiencia depende del modelo y especificación. En México están reguladas por la NOM-116-STPS-1994. Por su forma, la NOM los divide en respiradores de media cara que cubren nariz, boca y barbilla, y de cara completa, que incluye los ojos. ${ }^{15}$

Existen nueve tipos de respiradores desechables para partículas que cubren nariz, boca y barbilla aprobados. Se clasifican con base en su eficiencia de filtración y resistencia a aceites. En el área médica se utilizan los $\mathrm{N}$ que no resisten aerosoles de aceite (N95, N99, N100). La OMS recomienda el uso de respiradores N95 o superiores que filtran más del 95\% de las partículas de 0.1 a $0.3 \mu \mathrm{m}$, en pacientes con definición operacional de COVID-19. ${ }^{14}$

Existen diferentes modelos que varían en la forma, el material y la presencia de válvula de exhalación. El uso del respirador con válvula de exhalación es recomendable, ya que disminuye la sensación de sofocamiento y humedad, pero no debe utili- 
zarse en áreas estériles, ya que al exhalar, el aire es expulsado a través de la válvula al medio ambiente. Los respiradores con válvula y sin envoltura no se consideran resistentes a los fluidos y, por lo tanto, deben usarse con un protector facial completo si se anticipan salpicaduras de sangre o fluidos corporales. ${ }^{16,17}$

La forma de colocación es muy importante y debe realizarse de la siguiente forma:

1. Evitar maquillaje y no portar barba/bigote que pueda interferir con el sellado.

2. Verificar que no haya ruptura del equipo o de las cintas elásticas, que tenga el clip nasal en posición adecuada y que no esté manchado.

3. Colocación:

- Coloque el respirador sobre su nariz y boca, con el clip metálico hacia arriba.

- Jale la cinta superior y colóquela sobre la coronilla de la cabeza, arriba de las orejas.

- Coloque la cinta inferior debajo de las orejas.

- Moldee el clip metálico con ambas manos, asegurando el sellado alrededor de la nariz.

4. Corroborar sellado.

Para respiradores sin válvula, coloque ambas manos completamente sobre el respirador y exhale verificando que el respirador se abulte ligeramente, en el caso de los respiradores con válvula colocar las manos sobre el respirador, inhalar y percibir el colapso.

5. Evitar tocarse el respirador mientras lo tenemos puesto.

6. Desechar en caso de ruptura o falta de sello.

7. Desechar al término de la jornada o proceder al reuso/ descontaminación.

- Reúso de respiradores N95.

Dada la emergencia sanitaria, se ha reportado desabasto de respiradores N95, lo cual ha generado la necesidad de estrategias de reuso. Aunque no es una medida aprobada y sólo ha sido evaluada la descontaminación en ambientes controlados de SARS-CoV-2, debe considerarse en este momento de crisis para garantizar su disponibilidad continua. Estas estrategias en estudio son aplicables sólo para respiradores N95 y no están sugeridas para respiradores equivalentes. ${ }^{18}$

1. Se sugiere tener cinco respiradores, marcarlos del $1 \mathrm{al}$ 5, y usarlos uno cada día.

2. Al término del turno, retirar el respirador mediante las bandas elásticas sin tocar el frente y colocar en una bolsa, preferentemente de papel transpirable, y almacenarla.

3. Usar al siguiente día el respirador marcado \#2, al término de la jornada, y así sucesivamente, hasta terminar con el \#5, y reiniciar con el \#1.

4. Los respiradores deben guardarse y almacenarse en empaques individuales.

\section{- Descontaminación mascarilla N95.}

Existen tres métodos de descontaminación propuestos:

Peróxido de hidrógeno es el más prometedor, efecto mínimo en la filtración y el ajuste, con una eficacia del 99.9\% en la eliminación de esporas bacterianas, con una resistencia entre 20 a 50 ciclos de descontaminación. ${ }^{19}$

Radiación ultravioleta (UVGI) depende de la dosis. Lindsley y colaboradores, en su estudio sobre los efectos de la radiación ultravioleta en los respiradores N95, observaron diminución no significativa en el rendimiento de la filtración y esencialmente ningún efecto en la resistencia a dosis de hasta $950 \mathrm{~J} / \mathrm{cm}^{2}$, ciclos de desinfección estará limitado por el modelo de respirador y la dosis de UVGI requerida para inactivar el patógeno. ${ }^{19-22}$ En su estudio Heimbuch y su grupo determinaron que a dosis de $18 \mathrm{~kJ} / \mathrm{m}^{2}$ disminuye más de 4 log la concentración del virus de H1N1 sin deterioro o deformación del respirador.

Métodos de calor: Heimbuch y colaboradores describen tres diferentes métodos para descontaminar seis diferentes tipos de respiradores N95, introduciendo los respiradores en horno de microondas, horno de convección y rayos ultravioleta. Concluyendo que los tres métodos son efectivos para disminuir y desnaturalizar el virus en la superficie de los respiradores. Chen y su equipo en su estudio verificaron la descontaminación de los respiradores N95 de manufacturación China, ellos inocularon los respiradores con el virus H120 para simular SARS-CoV-2, ya que ambos son coronavirus, observando que a baño maría durante cinco minutos no se encontró carga viral en la superficie del respirador en $98 \%{ }^{23,24}$

Todo respirador que ha sido sometido a algún proceso de descontaminación deberá verificarse su adecuado funcionamiento, en caso contrario deberá desecharse. ${ }^{25}$

\section{Respiradores elastoméricos}

1. Tipo de máscara: máscara de media cara reutilizable.

2. Material: elastómero termoplástico (TPE).

Tabla 2: Ventajas de respiradores elastoméricos de media cara vs respiradores N95.

\begin{tabular}{|l|c|c|}
\hline Característica & $\begin{array}{c}\text { Elastomérico de } \\
\text { media cara }\end{array}$ & $\begin{array}{c}\text { Respirador } \\
\text { N95 }\end{array}$ \\
\hline Desechable & No & Sí \\
\hline Lavable & Sí & No \\
\hline Tiempo de uso & $\begin{array}{l}\text { Hasta 40 horas con- } \\
\text { tinuas 0 30 días con } \\
\text { horas discontinuas }\end{array}$ & $\begin{array}{l}\text { 8 horas de uso con- } \\
\text { tinuo o intermitente }\end{array}$ \\
\hline
\end{tabular}

* Dependiendo de su uso adecuado, del ambiente en donde se utilice y con los filtros que se utilice. ${ }^{24}$ 
3. Aprobado para protección respiratoria contra partículas, vapores orgánicos, cloro ácido clorhídrico, fluoruro de hidrógeno, dióxido de azufre, amoniaco metilaminas, formaldehído, radionucleidos (según los filtros que utilicen).

4. No usar en atmósferas cuyo contenido de oxígeno sea menor a $19.5 \%$ (Tabla 2).

Respirador purificador de aire por filtración a presión (PAPR por sus siglas en inglés)

Éste es un equipo que pasa el aire a través de un filtro de partículas de alta eficiencia (HEPA: High Efficiency Particulate Air) impulsado por un motor y llega al usuario por un tubo conectado a una capucha de polietileno de alta densidad, la cual debe ser de un solo usuario. Se debe mantener la pila del motor con carga adecuada. Los equipos mencionados sólo filtran el aire presente en el medio ambiente, por lo que si existe algún tipo de contaminante tóxico pasará a los usuarios. ${ }^{14,17}$

\section{Equipo de protección ocular}

El comportamiento de transmisión de COVID-19 se ha comparado con el de SARS y MERS. Aunque no hay evidencia de transmisión ocular en las cepas previas de SARS (2003) y MERS (2012), debe tomarse en cuenta que en SARS-CoV-2 puede ser una forma de transmisión, ya que la presencia de conjuntivitis se informó en un 4.0-5.5\%, y la secreción conjuntival en $9.0-10.5 \%$ de los pacientes con diagnóstico confirmado de COVID-19. ${ }^{26-31}$ En la actualidad, no hay evidencia directa en ensayos aleatorios de que el equipo de protección ocular por sí solo impida la transmisión de SARSCoV-2; sin embargo, se recomienda el uso al tener contacto directo con pacientes, recolección de muestras que pueden producir aerosoles oculares, cirugía ocular interna, etcétera. El uso de lentes de seguridad es obligatorio en cualquier personal que realice vigilancia o manejo de la vía aérea. ${ }^{28,30,32}$

De acuerdo al equipo con el que se cuente por unidad, puede tratarse de pantalla facial o lentes de seguridad. Los protectores faciales proporcionan cobertura facial completa. Las gafas brindan una excelente protección ocular, pero tienen la desventaja del empañamiento. .6-28,30-33 $^{-1}$

Los lentes de protección ocular deben hacer un buen sello contra la piel de la cara, tener marco de policarbonato o policloruro de vinilo (PVC) flexible para encajar fácilmente con todos los contornos de la cara con presión uniforme, hermético en los ojos y las áreas circundantes. Debe ser ajustable para los usuarios de anteojos graduados, y tener un lente de plástico transparente con tratamiento antiempañante, banda ajustable para asegurar firmemente que no se desajuste durante la actividad clínica y ventilación indirecta para evitar el empañamiento. ${ }^{34,35}$
Las caretas y los lentes pueden ser reutilizables cuando su material sea de policarbonato o policloruro de vinilo, siempre que existan disposiciones apropiadas para la descontaminación. Debe asegurarse que el material cuente con características recomendadas por la industria y puede tolerar hasta más de 25 lavados con alcohol, solución alcalina y agua sin perder la flexibilidad y propiedades antiempañamiento. ${ }^{34,35}$

Para la desinfección, se puede utilizar solución clorada en una concentración de cinco partes por millón $(95 \mathrm{~mL}$ de cloro al 6\% para 1 litro de agua) o alcohol al 70\%.34,35

Al remover el equipo, de acuerdo con los pasos establecidos para retiro de EPP, es importante evitar el contacto con la superficie externa del equipo de protección y retirar el mismo tomando las cintas y jalando hacia arriba y adelante. Se recomienda colocar en un área designada para desinfección en caso de ser material reutilizable. ${ }^{26}$

El espectro clínico de COVID-19 es amplio, presentándose desde casos asintomáticos hasta presentaciones severas, principalmente en edades avanzadas y en pacientes con comorbilidades. En algunas situaciones, los pacientes requerirán soporte crítico y con ello exposición a PGA, lo que incrementa el riesgo de trasmisión de la enfermedad, en especial en trabajadores de la salud. ${ }^{33,36,37}$

Con la finalidad de disminuir el riesgo de transmisión del SARS-CoV-2 durante los PAG, principalmente durante la intubación endotraqueal, se consideran las siguientes pautas.

\section{Intubación endotraqueal \\ Consideraciones generales}

1. Se sugiere uso de EPP para los trabajadores de la salud que intubarán a un paciente que cumple con la definición operacional. Se recomienda el uso de bata adicional o mandil plástico. ${ }^{38}$

2. Se recomienda una intubación temprana y programada en un ambiente controlado de la mejor manera posible. ${ }^{38}$

3. La técnica elegida puede diferir de acuerdo con las prácticas y equipos locales, así como la disponibilidad de insumos en cada centro hospitalario.

4. Idealmente, el procedimiento de intubación debe realizarse en sala aislada con presión negativa (con al menos 12 cambios de aire/hora). ${ }^{39}$

5. Se debe realizar por el personal médico más experto, los intentos múltiples aumentan el riesgo para el paciente y el personal expuesto. ${ }^{38}$

6. Siempre que esté disponible un videolaringoscopio y personal capacitado en su uso, se recomienda su utilización, para maximizar la distancia entre el operador y la orofaringe del paciente. 
7. Se recomienda evitar la intubación con broncoscopio de fibra óptica y el paciente despierto, salvo que sea estrictamente necesario debido a una anatomía anormal de las vías respiratorias. En caso de realizarse, utilice equipos flexibles desechables. ${ }^{38}$

8. Personal involucrado:

- Líder del equipo: maneja la vía aérea (el que va a intubar).

- Asistente del intubador: aplica presión cricoidea.

- Enfermera: aplica medicamentos, monitorea al paciente.

- Un circulante: se encuentra fuera de la sala, ayuda en caso necesario.

9. El equipo debe comunicarse con instrucciones simples, en voz alta. ${ }^{39,40}$

10. Preparar el equipo necesario para la instrumentación de la vía aérea y su manejo (todos los dispositivos acorde a la edad del paciente), así como los medicamentos apropiados fuera de la habitación. ${ }^{41}$

11. Se recomienda realizar una evaluación meticulosa de la vía aérea del paciente (indagar sobre posibles malformaciones de la vía aérea, test de Mallampati, cuello: corto-rígido, movimiento de la columna vertebral, antecedente de apnea obstructiva del sueño). ${ }^{41,42}$

12. En niños, la vía aérea puede clasificarse en tres tipos, se podrá complementar la evaluación con las escalas de predicción, si bien dichas escalas no se crearon de manera específica para la edad pediátrica, se han utilizado como herramienta de evaluación de vía aérea en edad pediátrica. ${ }^{43,44}$

13. El personal debe conocer los planes alternos a seguir en caso de presentarse dificultades para la intubación (Tabla 3). ${ }^{42}$

\section{Secuencia de intubación rápida}

a. Preoxigene al paciente con oxígeno al $100 \%$ con mascarilla reservorio durante cinco minutos o con puntas nasales al menos a $5 \mathrm{~L}$ por minuto durante cinco minutos. No se recomiendan dispositivos de alto flujo; sin embargo, si el paciente ya está usándolo, puede dejarse encendido durante la preoxigenación y la intubación, ya que esto evita mayor deterioro clínico. ${ }^{40}$

b. Verifique que el sujeto tenga colocada la mascarilla quirúrgica, la cual se retirará hasta el momento de la laringoscopia.

c. Si requiere ventilación con presión positiva, deberá colocar el filtro antibacteriano/viral en la bolsa autoinflable y haciendo un adecuado sello con técnica en E/V (no retire la mascarilla quirúrgica). Mantenga la ventilación con presión positiva con volúmenes pequeños. ${ }^{40}$

d. De preferencia, utilice tubo endotraqueal (TET) con globo, ya que éstos disminuyen los aerosoles.

e. Administre los medicamentos conforme a las guías de secuencia rápida de intubación. ${ }^{40-45}$

f. Asegúrese de que el bloqueo neuromuscular ocurra antes de la intubación, ya que esto elimina el reflejo de tos y con ello minimiza la aspersión de aerosoles.

g. La laringoscopia debe realizarse con el dispositivo con el que más fácil se logre la intubación traqueal al primer intento, manteniéndose tan alejado de la vía aérea como sea posible.

h. No ventilar y pinzar el tubo endotraqueal hasta su conexión con el ventilador. Algunas guías sugieren la colocación de gasas húmedas en el interior de la boca. ${ }^{39}$

i. Corrobore la posición del TET con la expansión bilateral de la pared torácica, si dispone de ello puede utilizar

Tabla 3: Signos predictores de vía aérea difícil en pediatría ${ }^{42,43}$

\begin{tabular}{|c|c|}
\hline $\begin{array}{c}\text { VAD } \\
\mathbf{P}\end{array}$ & $\begin{array}{l}\text { Vía área probable o sospechosa } \\
\text { Patología aguda: infecciones, trauma, cuerpos extraños, alergias } \\
\text { Padecimientos crónicos: asma, mal manejo de secreciones }\end{array}$ \\
\hline $\begin{array}{c}\text { VAD } \\
\text { I }\end{array}$ & $\begin{array}{l}\text { Vía área inesperada } \\
\text { Se presenta de forma aguda durante la intubación } \\
\text { Causas anatómicas: obstrucción de tejidos blandos } \\
\text { Inadecuada posición de la cabeza } \\
\text { Causas funcionales: secreciones, laringoespasmo }\end{array}$ \\
\hline$E$ & $\begin{array}{l}\text { Escalas de predicción } \\
\text { Distancia esternomentoniana: }<12 \mathrm{~cm} \\
\text { Escala de Mallampati: clase III-IV } \\
\text { Escala de Patil-Aldreti (distancia tiroideo-mentoniana): }<6.5 \mathrm{~cm} \text {, clase II-III } \\
\text { Extensión de la articulación atlanto-occipital: grado II. Si la extensión se limita en un tercio de su valor normal }\left(22^{\circ}\right)\end{array}$ \\
\hline
\end{tabular}


capnógrafo o estetoscopio con wireless. No ausculte directamente en tórax (Figura 1). ${ }^{40}$

1. Verifique los signos predictores de vía aérea difícil en pediatría.

2. Dependerá de la edad del paciente, no se recomienda cuando existe hipercapnia, inestabilidad hemodinámica y falla multiorgánica.

3. El material desechable no utilizado deberá de meterse en una bolsa de plástico y desecharse en el contenedor de residuos peligrosos biológicos infecciosos (RPBI).

4. Tubo endotraqueal.

\section{Durante la ventilación mecánica}

Filtros antivirales para dispositivos de presión positiva, bolsa autoinflable y ventiladores mecánicos en COVID-19.

- Las bolsas de presión positiva o autoinflables deberán usarse con un filtro antibacterial o antiviral. ${ }^{46,47}$

- Tiene el objetivo de filtrar los gases respiratorios y disminuir el contagio del personal de salud en la secuencia de intubación orotraqueal, así como en el ventilador mecánico en la rama inspiratoria y espiratoria. ${ }^{48-50}$

- Los filtros se deben cambiar con una periodicidad de 48 horas. $^{51}$

- Pueden ser de barrera o antielectrostáticos, ambos cumplen con la función de filtrar los gases. ${ }^{52}$

\section{Aspiración de secreción y cuidados del tubo endotraqueal (TET)}

- La aspiración de secreciones se encuentra dentro de los PAG, su importancia radica en que al ser una exposición estrecha (menor a $1.8 \mathrm{~m}$ ) con una persona infectada con generación de gotas (aerosoles grandes), que pueden depositarse en boca, nariz, ojos de la persona expuesta o bien inhalarse si se encuentra cerca en el momento de la expulsión de la misma. ${ }^{7,53,54}$

- Por dichas razones, el personal a cargo de esta actividad deberá contar con el EPP adecuado.55

- Usar el sistema de aspiración cerrado de secreciones, en caso de no contar con éste se sugiere el empaquetamiento de la boca con gasas. ${ }^{48,56}$

- Evitar las desconexiones, en caso de requerirlo, deberá poner el ventilador en pausa (stand by) y de manera simultánea pinzar el TET, el cual se despinzará hasta que el paciente sea reconectado nuevamente. ${ }^{46,57}$

- Se ha sugerido el uso de cánulas con globo, en caso de no contar con ellas y la fuga no sea evitable, considerar desde el inicio el empaquetamiento de la boca con gasas.

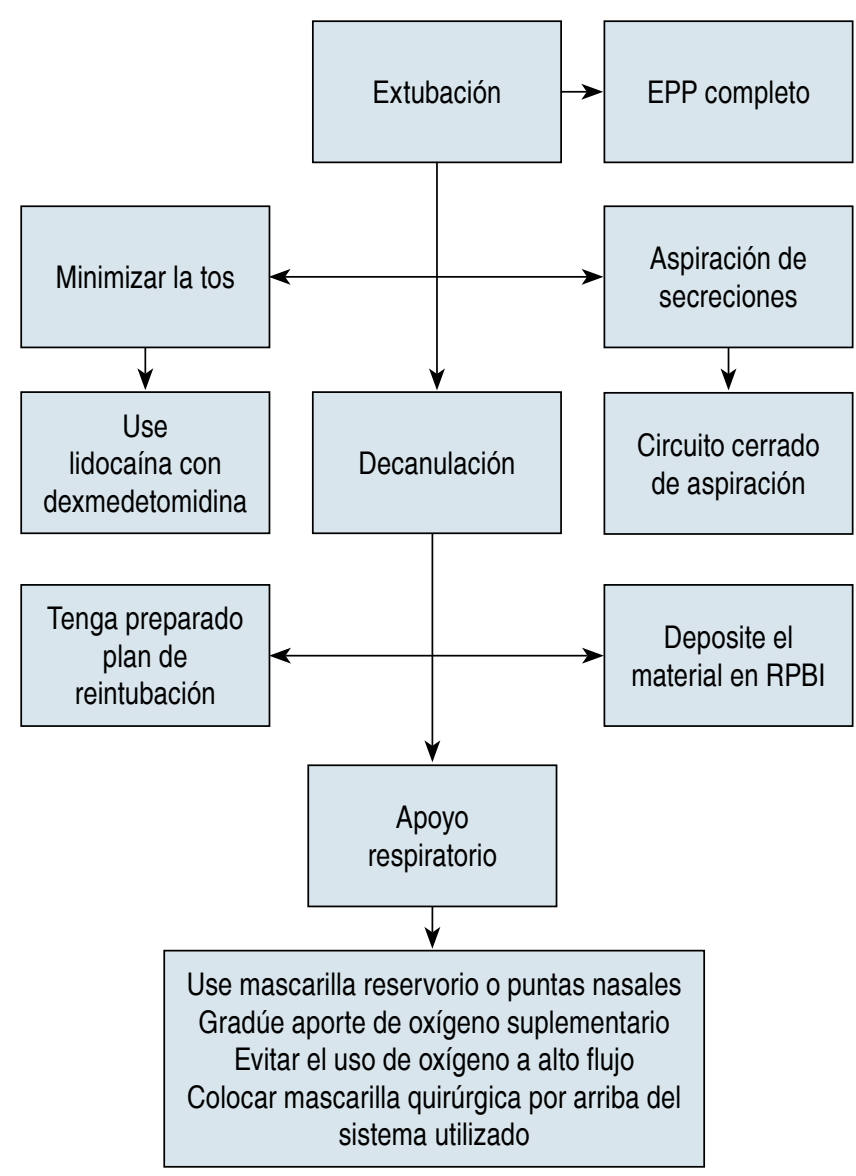

Figura 1: Algoritmo de extubación en pacientes con COVID-19.38-45 $\mathrm{EPP}=$ equipo de protección personal; $\mathrm{RPBI}=$ Residuos peligrosos biológicos infecciosos.

- Mantener una sedación y relajación adecuada para evitar al máximo estimulación que desencadene esfuerzo tusígeno del paciente, sobre todo al realizar procedimientos como la aspiración, movilización y fisioterapia. ${ }^{7,50-57}$

- En toda manipulación del paciente se sugiere el pinzamiento del TET.

\section{Humidificación}

- Evita humidificación activa y sistemas de calefacción para evitar condensación de los tubos. ${ }^{54}$

- Usar humidificación pasiva (circuito seco) con los dispositivos con los que se cuenten en sus unidades, a través de un conector estándar de $15 \mathrm{~mm}$ en el tubo endotraqueal, colocado en línea entre la pieza en Y del circuito de respiración y TET. ${ }^{58}$

- Mantienen la capacidad de humidificar hasta cuatro días con un cambio mínimo en la resistencia.

- Disminuye el riesgo de neumonía asociada al ventilador. ${ }^{60}$ 
- La eficiencia de filtración es del 99.9\% y eficiencia de humidificación de $>30 \mathrm{mg} / \mathrm{L.}^{52}$

\section{Sedación y relajación}

- Es prioritario mantener sedación y relajación adecuadas para evitar al máximo los esfuerzos tusígenos, sobre todo al realizar PAG.

- Fentanil o remifentanil son útiles al suprimir reflejos laríngeos.

- Lidocaína suprime el reflejo de tos. ${ }^{37}$

- La comunicación con anestesiología debe establecer para optimizar recursos y perfeccionar tratamientos durante la ventilación.

\section{Aerosolterapia}

- Evitar la terapia de inhalación, en caso necesario se prefieren los inhaladores de polvo seco y los inhaladores de dosis medida..$^{59}$

- En ventiladores mecánicos, se recomienda dispositivo de malla vibratoria.

- No es necesario retirar todos los dispositivos de aerosolterapia conectados a los circuitos de ventilación, recuerde que debe minimizarse la desconexión de los circuitos.

- Existen dos tipos de espaciadores, los rígidos de entrega de inhaladores de dosis medida (IDM) y los flexibles que tienen como ventaja disminuir el espacio muerto al ser plegables. ${ }^{60-62}$
- Los adaptadores de IDM son dispensadores de entrega intermitente de aerosoles entregadas en dosis medidas. Para uso en conexión en $\mathrm{Y}^{63}$

- En pacientes con respiración espontánea, utilizar dispositivos activados por inhalación (como inhaladores de polvo seco) o dispositivos de IDM y cámara espaciadora. Después de la inhalación, limpie la boquilla y la cámara espaciadora con una solución al 75\% de alcohol.

\section{Extubación}

\section{Objetivos:}

- Minimizar la aerosolización de patógenos.

- Minimizar la exposición de patógenos a la vía aérea del personal de salud.

- Minimizar la tos y la exposición a las secreciones. ${ }^{40,64}$

\section{Puntos clave:}

- Recuerde colocar mascarilla quirúrgica posterior a la extubación.

- No se recomienda terapia de alto flujo, ventilación no invasiva y nebulizaciones (Tabla 4).40,64

En pacientes con diagnóstico de COVID-19 que requieran procedimiento quirúrgico, se recomienda al momento de la extubación utilizar la técnica de máscara sobre tubo. ${ }^{65}$ La cual consiste en ocupar un segundo filtro de alta eficiencia, sellado con técnica de dos manos, cerrar la válvula de anestésicos inhalados, desinflar globo y des-

Tabla 4: Pasos durante la extubación. ${ }^{42,65,66}$

\begin{tabular}{|c|c|c|}
\hline Preparar & Procedimiento & Posteriormente \\
\hline $\begin{array}{l}\text { Estrategia de extubación } \\
\text { (incluido plan de reintubación) }\end{array}$ & Pinzar u ocluir cánula orotraqueal & \\
\hline $\begin{array}{l}\text { Definir plan de apoyo respiratorio después de } \\
\text { la extubación }\end{array}$ & Extube & \\
\hline $\begin{array}{l}\text { Equipo esencial: } \\
\text { 1. Dispositivos de bajo flujo } \\
\text { 2. Cubrebocas para paciente } \\
\text { 3. Equipo de reintubación } \\
\text { 4. Antiemético } \\
\text { 5. Para suprimir tos considere: dexmede- } \\
\text { tomidina, lidocaína u opioides }\end{array}$ & $\begin{array}{l}\text { Coloque el dispositivo de oxígeno de bajo } \\
\text { flujo (mascarilla reservorio o puntas nasales) } \\
\text { Coloque la mascarilla quirúrgica } \\
\text { Encienda el flujo de oxígeno a } 5 \text { litros por } \\
\text { minuto (titular posteriormente para mantener } \\
\mathrm{SaO}_{2} \text { mayor a } 92 \% \text { ) }\end{array}$ & \\
\hline
\end{tabular}




\begin{tabular}{|l|}
\hline \multicolumn{2}{|c|}{ Algoritmo de intubación en pacientes } \\
\hline \multicolumn{2}{|c|}{ Preparación previa } \\
\hline \multicolumn{2}{|c|}{ Preparación del equipo/verificación } \\
\hline \multicolumn{2}{|c|}{$\mid$\begin{tabular}{l|l|}
\hline \multicolumn{2}{|c|}{ Tener preparado un kit de intubación que pueda trasladarse } \\
\hline $\begin{array}{l}\text { 1. Laringoscopio o } \\
\text { videolaringoscopio }\end{array}$ & $\begin{array}{l}\text { 7. Fijadores para tubo } \\
\text { endotraqueal }\end{array}$ \\
\hline $\begin{array}{l}\text { 2. Tubo endotraqueal } \\
\text { 8. Gasas estériles, solución } \\
\text { salina y tela adhesiva }\end{array}$ \\
\hline $\begin{array}{l}\text { 3. Mascarillas laríngeas } \\
\text { 4. Bolsa y mascarilla con filtro } \\
\text { para virus/bacterias }\end{array}$ & $\begin{array}{l}\text { 9. Medicamentos para } \\
\text { sedación y relajación }\end{array}$ \\
\hline 5. Tubos para aspiración & caja de acrílico de \\
\hline 6. Jeringas & Monitor con oxímetro \\
\hline
\end{tabular}}
\end{tabular}

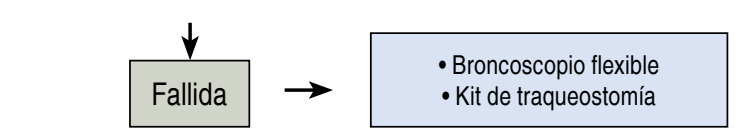

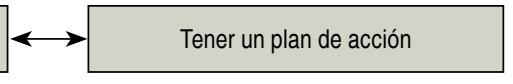

Realizar un simulacro y practicar el procedimiento

Máximo 3 personas dentro de la sala:

A. Líder: persona más experimentada, a cargo de vía aérea

B. Asistente: apoyo asistencia, experiencia en vía aérea plan $B$

C. Enfermera: administra medicamentos y verificar monitorización.

D. Una persona fuera de la sala: asistirá en caso de emergencia

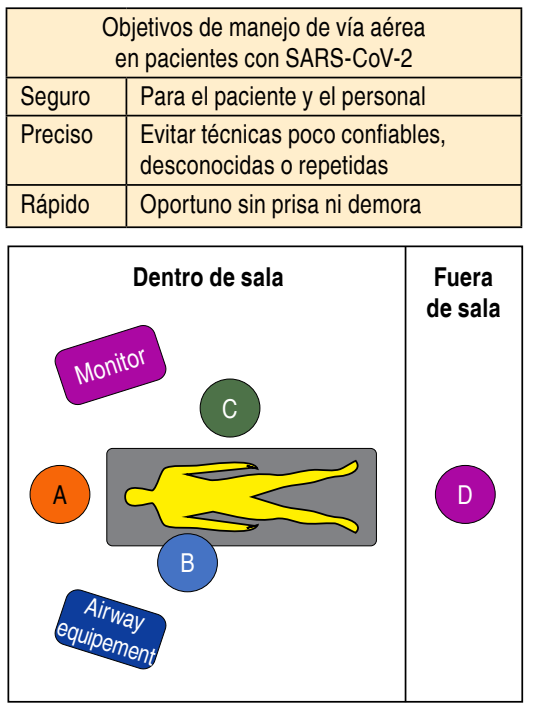

Caso sospechoso o confirmado de COVID-19

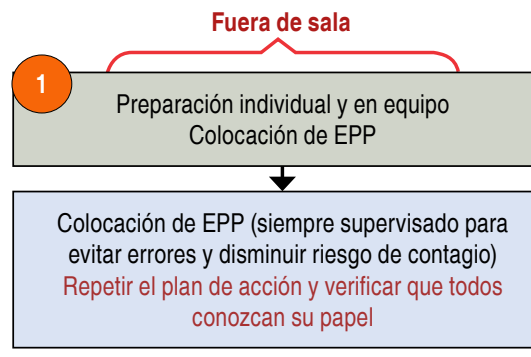

Secuencia rápida de intubación Considere:

Fentanil: $3-5 \mu \mathrm{g} / \mathrm{kg} / \mathrm{do}$

Propofol: $2 \mathrm{mg} / \mathrm{kg} / \mathrm{do}$

Rocuronio: $0.6-1.3 \mathrm{mg} / \mathrm{kg} / \mathrm{do}$

Vecuronio: $50-100 \mu \mathrm{g} / \mathrm{kg} / \mathrm{do}$

Midazolam: $100-300 \mu \mathrm{g} / \mathrm{kg} / \mathrm{do}$

Detente, piensa, comunícate, PIDE AYUDA Ventilación con técnica en $\mathrm{V}$ (2 personas) Después de 3 intentos fallidos considerar:

Dispositivo de rescate: mascarilla laríngea de segunda generación

$$
\neg \text { Falla }
$$

* Broncoscopio flexible * Kit de traqueostomía



Dentro de sala

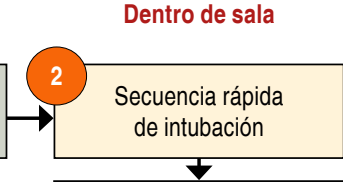

Posicionar al paciente en olfateo

Verificar acceso i, v y monitorización

Verificar posibilidad de vía aérea difícil
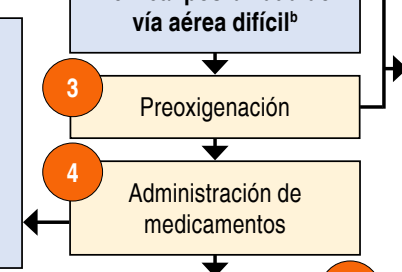

Administración de medicamentos

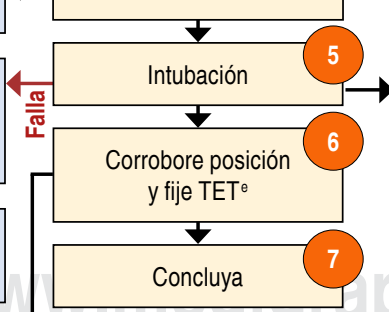

Visualizando movimientos del tórax capnografía, estetoscopio inhalámbrico, oximetría y radiografía

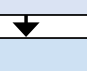

Si debe permanecer en sala retírese la segunda bata o el mandil plástico, retire segundo par de guantes y cálcese uno nuevo ${ }^{\dagger}$

Indique la desinfección de la sala después de 20 minutos de haber realizado el procedimiento

Figura 2: Algortimo de Intubación en pacientes con COVID-19.38-45

a = El uso de las cajas de acrílico es controversial y no está estandarizado; b = Consulte Tabla 3: Signos predictores de vía aérea difícil en pediatría; $c=$ Dependerá de la edad del paciente, no se recomienda cuando existe hipercapnia, inestabilidad hemodinámica y falla multiorgánica; $d=$ El material desechable no utilizado deberá meterse en una bolsa de plástico y desecharse en el contenedor de Residuos peligrosos biológicos infecciosos (RPBI); e = Tubo endotraqueal. 
conectar el circuito. ${ }^{65,66}$ Asenjo describe una variación en esta técnica al sobreponer una cobertura plástica sobre la mascarilla, la cual se retira una vez que se corrobora que el paciente tiene una respiración estable y ausencia de tos. ${ }^{57,67}$ Numerosas técnicas con dispositivos novedosos se han descrito para minimizar la dispersión de aerosoles durante la extubación, desde cajas de acrílico, siendo éstas las más difundidas, bolsas plásticas e inclusive el uso de cajas de cartón. El objetivo de estas herramientas es adicionar una barrera de protección al EPP y disminuir la contaminación por aerosoles. Su uso deberá sujetarse a la disponibilidad de cada centro (Figura 2). ${ }^{56,67-69}$

\section{Reanimación cardiopulmonar}

Consideraciones generales:

1. Todo el personal de salud que trate pacientes con sospecha o confirmación de COVID-19 deben usar correctamente el EPP. ${ }^{70-72}$

2. El equipo de reanimación consta de tres personas: ${ }^{71}$

Persona 1: vía aérea.

Persona 2: compresiones torácicas.

Persona 3: administración de medicamentos.

Persona 4: miembro reserva (tiempo, apoyo logístico, supervisión de protección contra la infección, cambio de compresiones torácicas, desfibrilación).

3. La intubación endotraqueal debe ser realizada por personal experimentado. ${ }^{71,72}$

4. En caso de no estar disponible experto en vía aérea, realizar técnica bolsa-mascarilla para dos reanimadores (uno sella la vía aérea y el otro ventila), con filtro bacteriano/viral hasta que llegue alguien capacitado para manejo avanzado de la vía aérea. Si está familiarizado, puede realizar este procedimiento utilizando la caja de acrílico para evitar mayor contacto con secreciones. ${ }^{71-73}$

5. La habitación debe ser limpiada y desinfectada al terminar el procedimiento. ${ }^{70}$

En caso de paro con un solo reanimador:

1. Iniciar compresiones torácicas, incluso en un paro hipóxico. Monitorice el ritmo lo antes posible. ${ }^{73}$

2. Si el paciente ya está recibiendo terapia de oxígeno de bajo flujo o está conectado a un ventilador, manténgalos y continúe con compresiones torácicas.

3. Si hay duda sobre el diagnóstico de paro cardíaco, inicie las compresiones.

Evite en todo momento: ${ }^{71,72}$

1. Colocar su oreja o mejilla cerca de la boca del paciente.

2. Ventilar boca a boca o usar mascarilla de bolsillo.
Vía aérea en reanimación neonatal: $: 74,75$

1. Las últimas recomendaciones sugieren no limpiar las vías respiratorias a través de succión si no es necesario, ya que esto podría generar aerosoles.

2. Si el paciente requiere ventilación, el personal más experimentado debe realizar la intubación.

\section{CONCLUSIONES}

La pandemia de COVID-19 ha colocado a la bioseguridad como elemento clave en el tratamiento de estos pacientes, que hoy en día era en gran parte desconocida para el personal de salud. Las medidas de protección en situaciones generadoras de aerosoles en COVID-19 sin duda modificarán a largo plazo la práctica en los diferentes escenarios de atención en salud de nuestro país y del mundo. La perfección de las mismas disminuirá sobremanera la posibilidad de contagio. La generación de normas antes, durante y después de la ventilación invasiva resulta fundamental para el tratamiento apropiado de los pacientes, las cuales permiten una actuación certera en la práctica del equipo, quienes deben trabajar de manera coordinada para limitar el contagio en los trabajadores de la salud.

\section{REFERENCIAS}

1. Organización Mundial de la Salud. Bioseguridad y bioprotección [Internet]. 2018 [Consultado 30 de marzo de 2020]. Disponible en: https:// www.who.int/influenza/pip/BiosecurityandBiosafety_ES_20Mar2018.pdf

2. Organización Mundial de la Salud. Manual de bioseguridad en el laboratorio. 3a ed. [Internet]. 2004 [Consultado marzo de 2020]. Disponible en: http://www.who.int/csr/resources/publications/biosafety/ CDS_CSR_LYO_2004_11SP.pdf

3. Richmond JY, McKinney RW. Bioseguridad en laboratorios de microbiología y biomedicina. 4⿳亠丷a edición. Bethesda, Maryland: CDC Library collection; 2002.

4. Organización Mundial de la Salud. Rational use of personal protective equipment for coronavirus disease (COVID-19): interim guidance [Internet]. 2020 [Consultado 30 de marzo de 2020]. Disponible en: https://apps.who.int/iris/handle/10665/331215

5. Ferioli M, Cisternino C, Leo V, Pisani L, Palange P, Nava S. Protecting healthcare workers from SARS-CoV-2 infection: practical indications. Eur Respir Rev 2020;29:200068. doi: 10.1183/16000617.0068-2020.

6. Cook T. Personal protective equipment during the COVID-19 pandemic - a narrative review. Anaesthesia 2020. doi: 10.1111/anae.15071.

7. Tran K, Cimon K, Severn M, Pessoa-Silva CL, Conly J. Aerosol generating procedures and risk of transmission of acute respiratory infections to healthcare workers: a systematic review. PLoS ONE 2012;7(4):e35797. doi: 10.1371/journal.pone.0035797.

8. European Centre for Disease Prevention and Control. Infection prevention and control for COVID-19 in healthcare settings - Third update [Internet]. [Consultado 7 de abril de 2020] ECDC: Estocolmo; 2020.

9. Vázquez L, Cortés L, León F, Flores F, López P, et al. Niveles de bioseguridad y manejo de la vía aérea en pacientes con infecciones 
tipo neumonía por COVID-19. Reporte especial COVID-19. Sociedad Científica Internacional EMIVA [Internet]. 2020 [consultado 15 de abril de 2020]. Disponible en: https://drive.google.com/file/d/13JoDKXntG F1CAoFzoAgTCHbQRhBObfEj/view

10. Public health England Recommended PPE for health e care workers by secondary care in patient clinical setting, COVID-19 personal protective equipment PPE. NHS [internet]. 2020 [Consultado el 12 de abril de 2020]. Disponible en: https://assets.publishing.service.gov.uk/ government/uploads/system/uploads/attachment_data/file/879107/ T1_poster_Recommended_PPE_for_healthcare_workers_by_secondary_care_clinical_context.pdf

11. Instructivo sobre uso de mascarillas: triple capa, quirúrgica tipo concha y respirador N95 [Internet]. 2020 [Consultado el 28 de abril de 2020]. Disponible en: http://educacionensalud.imss.gob.mx/es/ system/files/Instructivo_Uso_mascarillas\%20y\%20respiradores\%20 N95_DPM_09.04.2020_19h.pdf

12. Li L. Protection for medical staff. Care of pediatric patients during COVID-19 pandemic [Internet]. 2020 [Consultado el 13 de abril de 2020]. Disponible en: https://drive.google.com/file/d/1hG09QRQMFd89PHYNUgiybp2TUAual2e/view

13. St Paul MN U. 3M science applied to life. Comparison of FFP2, KN95 and N95. Filtering facepiece respirator classes [Internet]. 2020 [Consultado el 12 de abril de 2020]. Disponible en: https://multimedia.3m. com/mws/media/17915000/comparison-ffp2-kn95-n95-filteringfacepiece-respirator-classes-tb.pdf

14. Torres-Hernández KJ, Sevilla-Reyes EE. Conceptos para la selección y uso de mascarillas y respiradores, como medidas de protección durante los brotes de influenza. Rev Inst Nal Enf Resp Mex 2009;22(3):230-237.

15. NOM-116-STPS-1994 Norma Oficial Mexicana, Seguridad-respiradores purificadores de aire contra partículas nocivas [Internet]. [Consultado 20 de abril de 2020] Disponible en: http://www.dof.gob. $\mathrm{mx} /$ normasOficiales/3926/stps3/stps3.htm

16. COVID-19 personal protective equipment (PPE) [Internet]. Inglaterra: Salud Pública: 2020 [Consultado 15 de abril de 2020]. Disponible en: https://www.gov.uk/government/publications/wuhan-novel-coronavirus-infection-prevention-and-control/covid-19-personal-protectiveequipment-ppe\#section-7

17. Sarabia MC. Uso de respiradores desechables de alta eficiencia para partículas. En: INER. Equipo de protección personal. [Internet]. 2009 [Consultado el 13 de abril de 2020]. Disponible en http://www.iner.salud. gob.mx/descargas/comitebioseguridad/ProteccionRespiratoria.pdf

18. Decontamination methods for $3 \mathrm{M}$ N95 respirators. Boletín Técnico [Internet]. 2020 [Consultado 15 de abril de 2020]. Disponible: https:// multimedia.3m.com/mws/media/18248690/decontaminationmethods-for-3m-n95-respirators-technical-bulletin.pdf

19. Descontaminación y reutilización de respiradores con máscara filtrante. Centers of Disease Control [Internet]. 2020 [Consultado 27 de abril de 2020]. Disponible en: https://www.cdc.gov/coronavirus/2019-ncov/ hcp/ppe-strategy/decontamination-reuse-respirators.html

20. Mills D, Harnish DA, Lawrence C, Sandoval-Powers M, Heimbuch BK. Ultraviolet germicidal irradiation of influenza-contaminated N95 filtering facepiece respirators. Am J infect Control 2018;46(7):e49-e55.

21. Qing-Xia M, Hu S, Chuan-Mei Z, Hong-Liang Z, Gui-Mei L, Rui-Mei $Y$, et al. Decontamination of face masks with steam for mask reuse in fighting the pandemic COVID-19: experimental supports. J Med Virol First 2020. Available from: https://doi.org/10.1002/jmv.25921

22. Lindsley WG, Martin SB Jr, Thewlis RE, Sarkisian K, Nwoko JO, Mead KR, et al. Effects of ultraviolet germicidal irradiation (UVGI) on
N95 respirator filtration performance and structural integrity. J Occup Environ Hyg. 2015;12(8):509-517. 10.1080/15459624.2015.1018518

23. Chen JM, Xia Q, Shan H. Decontamination of face mask with steam for mask reuse in fighting the pandemic COVID-19: experimental supports. J Med Virol 2020. doi: 10.1002/jmv.25921.

24. Heimbuch BK, Wallace WH, Kinney K, Lumley AE, Wu C-Y, Woo $\mathrm{M}-\mathrm{H}$, et al. A pandemic influenza preparedness study: Use of energetic methods to decontaminate filtering facepiece respirators contaminated with H1N1 aerosol and droplets. Am J Infect Control. 2011;39(1):e1-e9. doi: 10.1016/j.ajic.2010.07.004.

25. Technical Report for Heat-Humidity-Based N95 Reuse Risk Management. N95Decon Research [Internet]. 2020 [Consultado 27 de abril de 2020] Disponible en: https://static1.squarespace.com/static/5e8126f89327941b9453eeef/t/5e86d4b6c806 bd1197556052/1585894582592/200402_N95DECON_Heat_technicalreport_v1.2_final.pdf

26. Organización Mundial de la Salud. Requirements and technical specifications of personal protective equipment "PPE" for de novel coronavirus (2019-ncov) in healthcare settings [Internet]. [Consultado 23 de abril de 2020]. Available from: https://www.paho.org/en/ documents/requirements-and-technical-specifications-personalprotective-equipment-ppe-novel

27. Sommer A. Humans, viruses, and the eye- an early report from the COVID-19 front line. JAMA Ophthalmol 2020;138(5):578-579.

28. What is the efficacy of eye protection equipment compared to no eye protection equipment in preventing transmission of COVID19-type respiratory illnesses in primary and community care? The Centre for Evidence-Based Medicine develops, promotes and disseminates better evidence for healthcare. Universidad de Oxford [Internet]. [Consultado el 20 de abril de 2020]. Available from: https://www.cebm.net/covid-19/what-is-the-efficacy-of-eyeprotection-equipment-compared-to-no-eye-protection-equipmentin-preventing-transmission-of-covid-19-type-respiratory-illnessesin-primary-and-community-care/

29. Li JO, Lam DSC, Chen Y, Ting DSW. Novel Coronavirus disease 2019 (COVID-19): the importance of recognising possible early ocular manifestation and using protective eyewear. $\mathrm{Br} \mathrm{J}$ Ophthalmol 2020;104(3):297-298.

30. Chen L, Deng C, Chen X, Zhang X, Chen B, Yu H, et al. Ocular manifestations and clinical characteristics of 534 cases of COVID-19 in China: A cross-sectional study. medRxiv 2020. doi: https://doi. org/10.1101/2020.03.12.20034678

31. Ong SWX, Tan YK, Chia PY, Lee TH, Ng OT, Wong SY, et al. Air, surface environmental, and personal protective equipment contamination by severe acute respiratory syndrome coronavirus 2 (SARS-CoV-2) from a symptomatic patient. JAMA. 2020;323(16):1610-1612. 10.1001/ jama.2020.3227

32. Guía recomendada para uso extendido y reutilización limitada de respiradores con máscara filtrante N95 en entornos de atención médica. Centers for Disease Control and Prevention [Internet]. [Consultado el 27 marzo 2020]. Disponible en: https:/www.cdc.gov/niosh/topics/ hcwcontrols/recommendedguidanceextuse.html

33. Livingston $E$, Desai A, Berkwits M. Sourcing personal protective equipment during the COVID-19 pandemic. JAMA 2020. doi: 10.1001/ jama.2020.5317.

34. Doremalen N, Bushmaker T, Morris DH, Holbrook MG, Gamble A, Williamson BN, et al. Aerosol and surface stability of SARS-CoV-2 as compared with SARS-CoV-1. N Engl J Med. 2020;382:1564-1567. 10.1056/NEJMc2004973 
35. Sun CB, Wang YY, Liu GH, Liu Z. Role of the eye in transmitting human coronavirus: what we know and what we do not know. Research gate pre impression. doi: 10.20944/preprints202003.0271.v2.

36. Chen Y, Li L. SARS-CoV-2: virus dynamics and host response. Lancet Infect Dis 2020;20(5):515-516. doi: 10.1016/S1473-3099(20)30235-8

37. Meng L, Qiu H, Wan L, Ai Y, Xue Z, Guo Q, et al. Intubation and ventilation amid the COVID-19 Outbreak: Wuhan's experience. Anesthesiology. 2020;132(6):1317-1332. doi: 10.1097/ALN.0000000000003296

38. Zuo MH, Huang YG, Ma WH, Xuw ZG, Zhang JQ, Gong YH, et al. Expert recommendations for tracheal intubation in critically ill patients with novel coronavirus disease 2019. Chin Med Sci J 2020;35:e1-e9. doi: 10.24920/00372.

39. Cook TM, El-Boghdadly K, McGuire B, et al. Consensus guidelines for managing the airway in children with COVID-19; Highlighting differences in practice from adult guidelines. Anaesthesia [Internet]. [Consultado el 10 de abril de 2020] Disponible en: https://icmanaesthesiacovid-19.org/airway-management-guidance

40. Cook TM, El-Boghdadly K, McGuire B, McNarry AF, Patel A, Higgs A. Consensus guidelines for managing the airway in patients with $\mathrm{CO}$ VID-19: Guidelines from the Difficult Airway Society, the Association of Anaesthetists the Intensive Care Society, the Faculty of Intensive Care Medicine and the Royal College of Anaesthetists. Anaesthesia 2020;75(6):785-799. doi: 10.1111/anae.15054.

41. Difficult Airway Society: Paediatric Difficult Airway Guidelines [Internet]. [Consultad 09 de abril de 2020]. Disponible en: https://das. uk.guidelines/paediatric-difficult-airway-guidelines

42. Lipshaw J. Lista de verificación de manejo de vías aéreas sospechadas confirmadas COVID-19 [Internet]. [Consultado 09 de abril de 2020]. Disponible en: https://harvardmedsim.org/blog/covid19-airway-checklist-coronavirus/

43. Echeverry Marín PC, Engelhardt T. Algoritmo para el manejo de la vía aérea difícil en pediatría. Rev Colomb Anestesiol 2014;42(4):325-334.

44. Figueroa-Uribe F, Flores-del Razo JO, Vega-Rangel V, MéndezTrejo V, Ferrer-López M, González-Chávez NA. Escalas predictoras para identificar vía aérea difícil en población pediátrica: su utilidad en el servicio de urgencias. Rev Mex Pediatr. 2019;86(4):162-164. 10.35366/SP194H

45. Weingart S. COVID airway management thoughts. Blog de EMCrit. Publicado el 27/marzo/2020 [Internet]. [Consultado 09 de abril de 2020]. Disponible en: https://emcrit.org/emcrit/covid-airway-management

46. Calvo C, García LHM, De Carlos VJC. Recomendaciones sobre el manejo clínico de la infección por el "nuevo coronavirus» SARSCoV-2. Grupo de trabajo de la Asociación Española de Pediatría (AEP). An Pediatr (Barc) 2020;92(4):241.e1-241,e11

47. Mechanical ventilation of SARS patients. Safety issues involving breathing-circuit filters. Health Devices 2003;32(6):220-222.

48. Comité de Cuidados Respiratorios de la Sociedad Torácica China. Expert consensus on preventing nosocomial transmission during respiratory care for critically ill patients infected by 2019 novel coronavirus pneumonia. Zhonghua Jie He He Hu Xi Za Zhi 2020;17(0):E020.

49. Wax RS, Christian MD. Practical recommendations for critical care and anesthesiology teams caring for novel coronavirus (2019-nCoV) patients. Can J Anaesth 2020;67(5):568-576.

50. Lin LW, Hung TY. Swivel-HEPA-ETT (SHE) bougie and HEPA-ETT (HE) methods for safe intubation while managing patients with $\mathrm{CO}$ VID-19. Emerg Med J 2020;37(5):256-257.

51. Carrillo ER. Ventilación mecánica. Ciudad de México: Ed. Alfil; 2013. p. 488.
52. Fredes S, Gogniat E, Plotnikow G, Rodrigues-La Moglie R. Utilización de los filtros bacterianos/virales durante la ventilación mecánica invasiva. Medicina Intensiva 2013;30(1).

53. Hu VH, Watts E, Burton M, Kyari F, Mathenge C, Heidary F, et al. Protecting yourself and your patients from COVID-19 in eye care. Community Eye Health. 2020;33(108):S1-S6.

54. Documento técnico: Manejo clínico del COVID-19: unidades de cuidados intensivos del Ministerio de Sanidad, Sociedad Española de Pediatría, Sociedad Española de Urgencias de Pediatría, Sociedad Española de Cuidados Intensivos pediátricos, Sociedad Española de Infectología Pediátrica. Ministerio de Sanidad. Gobierno de España [Internet]. [Consultado 16 de abril de 2020]. Disponible en: https:// www.aeped.es/sites/default/files/protocolo_de_manejo_cla-nico_covid-19.pdf.pdf

55. Vázquez LA, Cortés LJA, León FOD, Flores FOA, López PCA. Bioseguridad y manejo de la vía aérea en el paciente critico -Puntos Clave-. Sociedad Científica Internacional [Internet]. [Consultado 14 de abril de 2020] Disponible en: https://fmcaac.net/website/wp-content/ uploads/2020/04/Reporte-Especial-EMIVA-COVID-19.pdf

56. Matava CT, Kovatsis PG, Lee JK, Castro P, Denning S, Yu J, et al. Pediatric airway management in COVID-19 patients: consensus guidelines from the Society for Pediatric anesthesia's pediatric difficult intubation collaborative and the Canadian Pediatric Anesthesia Society. Anesth Analg. 2020;131(1):61-73. doi: 10.1213/ ANE.0000000000004872.

57. Asenjo JF. Safer intubation and extubation of patients with COVID-19. Can J Anaesth 2020;1-3. doi: 10.1007/s12630-020-01666-9.

58. Plotnikow GA, Accoce M, Navarro E, Tiribelli N. Humidification and heating of inhaled gas in patients with artificial airway. A narrative review. Rev Bras Ter Intensiva 2018;30(1):86-97.

59. Cerpa F, Cáceres D, Romero-Dapueto C, Giugliano-Jaramillo C, Pérez R, Budini $\mathrm{H}$, et al. Humidification on ventilated patients: heated humidifications or heat and moisture exchangers? Open Respir Med J. 2015;9:104-111. 10.2174/1874306401509010104

60. Martínez FS, Maturana AD, Riquelme MH, Bustos GF, et al. Guía de recomendaciones para la implementación de soporte respiratorio pediátrico en COVID-19 [Internet]. [Consultado 12 de abril de 2020] Disponible: https://sochipe.cl/v3/covid/24.pdf

61. Ficha técnica Aerochamber MV. Trudell Medical International [Internet]. [Consultado el 31 de marzo de 2020] Disponible en: https://pdf. medicalexpo.es/pdf/trudell-medical-international-80196.html

62. Ficha técnica Aero Vent. Trudell Medical International [Internet]. [Consultado el 31 de marzo de 2020] Disponible en: https://pdf. medicalexpo.es/pdf/trudell-medical-international-80196.html

63. Ficha técnica conector para aerosol de MDI. Instrumentation Industries, Inc. [Internet]. [Consultado el 31 de marzo de 2020]. Disponible en: https://iiimedical.com/product-inserts/

64. The Australian and New Zealand Intensive Care Society. ANZICS. COVID-19 Guidelines. Melbourne: ANZICS [Internet]. [Consultado 09 de abril de 2020]. Disponible en: http://www.anzics.com.au/wp-content/ uploads/2020/03/ANZICS-COVID-19-Guidelines-Version-1.pdf.

65. Sydney Children's Hospitals Network. (s.f.). COVID-19 extubation checklist [Internet]. [Consultado 9 de abril de 2020]. Disponible en: https://www.nets.org.au/img.ashx?f=f\&p=covid_19\%2FCHW+COVI D+Extubation+Process+20032020.pdf

66. Tung A, Fergusson NA, Ng N, Hu V, Dormuth C, Griesdale DEG. Medications to reduce emergence coughing after general anaesthesia with tracheal intubation: a systematic review and network meta-analysis. $\mathrm{Br} J$ Anaesth 2020. doi: 10.1016/j.bja.2019.12.041. 
67. Patino Montoya M, Chitilian HV. Extubation barrier drape to minimise droplet spread. Br J Anaesth 2020;125(1):e195-e196. doi: 10.1016/j. bja.2020.03.028.

68. Lai YY, Chang CM. A carton-made protective shield for suspicious/ confirmed COVID-19 intubation and extubation during surgery. Anesth Analg 2020. doi: 10.1213/ANE.0000000000004869.

69. Canelli R, Connor CW, Gonzalez M. Barrier enclosure during endotracheal intubation. N Engl J Med 2020;382:1957-1958.

70. CPR \& Emergency Cardiovascular Care. Interim Guidance for Healthcare Providers during COVID-19 Outbreak. American Heart Association [Internet]. 2020 [Consultado el 31 de marzo de 2020]. Disponible en: https://professional.heart.org/idc/groups/ ahamahpublic/@wcm/@sop/@smd/documents/downloadable/ ucm_505872.pdf

71. Recomendaciones ante una parada cardíaca durante la pandemia de COVID-19. CCR Consell Català de Ressuscitación [Internet]. Marzo 2020 [Consultado el 31 de marzo de 2020]. Disponible en: https://www.semg.es/images/2020/Coronavirus/parada_cardíaca_ COVID-19.pdf
72. Recomendaciones en resucitación cardiopulmonar sobre COVID-19. Consejo de Resucitación del Reino Unido [Internet]. 10 marzo 2020 [consultado el 31 marzo 2020]. Disponible en: https:12//www.semes. org/wp-content/uploads/2020/03/23.-Rcp-paciente-COVID19.pdf

73. Pediatric Advanced Life Support. American Heart Association [Internet]. [Consultado el 30 de marzo de 2020]. Disponible en: https:// eccguidelines.heart.org/wp-content/themes/eccstaging/dompdfmaster/pdffiles/part-12-pediatric-advanced-life-support.pdf

74. Chandrasekharan P, Vento M, Trevisanuto D, Partridge E, Underwood MA, Wiedeman J, et al. Neonatal resuscitation and postresuscitation care of infants born to mothers with suspected or confirmed SARSCoV-2 infection. Am J Perinatol. 2020;37(8):813-824. doi: 10.1055/s0040-1709688.

75. Shalish W, Lakshminrusimha S, Manzoni P, Keszler M, Sant'Anna GM. COVID-19 and neonatal respiratory care: current evidence and practical approach. Am J Perinatol. 2020;37(8):780-791. doi: 10.1055/s-0040-1710522.

Conflicto de intereses: Los autores declaran no tener conflicto de intereses. 